\title{
Modelo Matemático da Pirólise do Etano em Forno Tubular
}

A. P. SPILIMBERGO, Departamento de Ciências Exatas e Engenharias, Universidade Regional do Noroeste do Estado do Rio Grande do Sul - UNIJUÍ, Caixa Postal 560, 98700-000 Ijuí, RS, Brasil.

V.G. KRIUKOV e A.V. FAFURIN, Departamento de Motores de Automóveis, Universidade Estatal Técnica de Kazan - UETK, Caixa Postal 420111, Rua K. Marx 10, Kazan, Rússia.

\begin{abstract}
Resumo. Neste trabalho é proposto um modelo matemático para o processo de pirólise do etano em forno tubular de serpentina. O meio reagente é descrito por um mecanismo constituído por 19 espécies e 63 reações químicas. É considerada a alteração da pressão, da temperatura e da velocidade do fluxo. Para resolver o sistema de equações resultante é utilizado o método de spline-integração com o esquema implícito. As simulações numéricas executadas para as condições de um forno industrial mostraram boa concordância com dados experimentais. Os resultados computacionais prevêem corretamente os fenômenos físico-químicos observados no forno e o modelo desenvolvido pode ser utilizado para projetá-lo.
\end{abstract}

Palavras-chave. Etano, Pirólise, Modelo Matemático, Forno Tubular, Mecanismo de Reações.

\section{Nomemclatura}

$T$ - temperatura; $P$ - pressão; $x$ - coordenada ao longo da tubulação; $w$ - velocidade do fluxo; $r_{i}$ - fração molar da $i$-ésima espécie do meio reagente; $T_{s}$ - temperatura externa da parede; $x_{f}$ - comprimento total do tubo; $\lambda_{g}$ - condutibilidade térmica do gás; $\eta_{g}$ - viscosidade do gás; $k_{j}$ - constante de velocidade da $j$-ésima reação; $n_{c}$ e $m_{c}$ - números de espécies e reações no meio reagente; $\mu_{i}$ - massa molecular da $i$-ésima espécie; $R_{o}$ - constante universal de gás; $Q_{m}$ - calor transferido à unidade de massa do gás a medida que ele se move pelo tubo; $\dot{m}$ - vazão total (reagente + vapor); $D$ - diâmetro interno do tubo; $\alpha_{\Sigma}$ - coeficiente total de transferência de calor ao fluxo; $R e, \operatorname{Pr}$ e $N u$ - números de Reynolds, Prandtl e Nusselt; $\mu_{g}$ massa molecular média do gás; $h_{o}^{*}$ - entalpia mássica de estagnação dos reagentes; $H_{q}^{a p}$ e $C_{q}^{a p}$ - entalpia e calor específico molar de referência da $q$-ésima espécie; $T_{a p}$ - temperatura de referência; $\xi$ - coeficiente de resistência hidráulica; $g_{e v}$ - relação mássica $\left(\mathrm{C}_{2} \mathrm{H}_{6} / \mathrm{H}_{2} \mathrm{O}\right)$ na entrada do forno; "o" e " $f$ " - índices dos estados inicial e final.

\section{Introdução}

O processo de pirólise do etano $\left(C_{2} H_{6}\right)$ é utilizado na petroquímica para produzir o etileno $\left(C_{2} H_{4}\right)$, que é o principal componente na produção do polietileno ([1], 
[2] e [11]). Para isso existem grandes plantas industriais e a pirólise ocorre em um forno tubular de radiação (Fig. 1), constituído por: tubo de serpentina (1), tela irradiante (2) e maçaricos de gás (3).

A taxa de decomposição do $C_{2} H_{6}$ depende do tempo de residência, da pressão e da temperatura. Quanto maior a parcela de etileno $\left(\mathrm{C}_{2} \mathrm{H}_{4}\right)$ nos produtos de pirólise, mais alto é o rendimento da planta. Assim, um problema atual é a criação de modelos matemáticos para prever a pirólise do $C_{2} H_{6}$ nestas instalações. Numerosos estudos são dedicados a esta temática ([1], [2], [3], [4] e [9], etc.).

Em [2] foi desenvolvido um modelo de pirólise em forno tubular considerando: a transferência de calor, a pirólise térmica e a precipitação de coque na parede. Este modelo leva em conta que a temperatura externa da parede é constante e utiliza um mecanismo simplificado de reações resolvidas pelo método de otimização dinâmica. Em [9], para modelar a pirólise do etano, é aplicada a "fuzzy possibilitic optimization". A conversão do etano em etileno é considerada em função da relação $g_{e v}$, da $T_{o}$ e da $P_{o}$. Mas, como considera o equilíbrio químico, os produtos de pirólise na saída do forno apresentam erros consideráveis. Um modelo de pirólise em forno tubular foi desenvolvido em [4] e nele é levado em conta a pirólise, a transferência de calor (por radiação e por convecção) e as perdas de pressão. Este modelo utiliza a abordagem de "reações estacionárias". No presente trabalho é desenvolvido um modelo deste tipo, mas considerando a cinética química detalhada.

\section{Esquema dos Processos e Equações Governantes}

Os reagentes gasosos $\left(\mathrm{C}_{2} \mathrm{H}_{6}+\mathrm{H}_{2} \mathrm{O}+2, \ldots, 5 \% \mathrm{CH}_{4}\right)$ com parâmetros: temperatura $(T \approx 800 \mathrm{~K})$, pressão $(P \approx 0.2, \ldots, 0.5 \mathrm{MPa})$ e relação $g_{e v} \approx 1, \ldots, 2$, entram no forno onde são aquecidos (até $T \approx 1200 \mathrm{~K})$ e o etano se decompõe. A tubulação $(D \approx$ $0.15 \mathrm{~m}$ ) inclui 8 a 10 trechos retos $(\approx 9 \mathrm{~m}$ cada) e pedaços curvos. Uma parcela do vapor de água é incluída no fluxo para reduzir a geração de coque nos produtos de pirólise. O aquecimento do tubo ocorre por irradiação das telas 2, as quais são aquecidas pelos maçaricos 3 . No andamento da pirólise ocorrem diferentes reações químicas, principalmente endotérmicas ([1] e [3]).
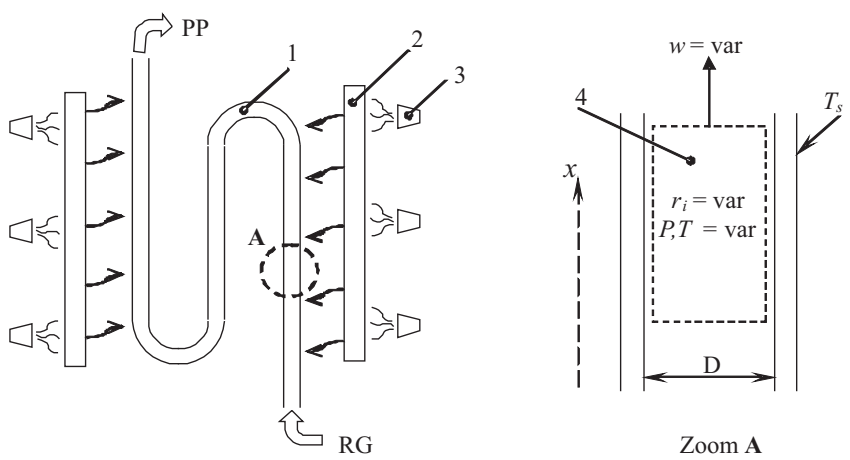

Figura 1: Esquema do forno tubular de radiação. 1 - tubo de serpentina; 2 - tela irradiante; 3 - maçaricos de gás; 4 - reator do deslocamento ideal; RG - reagentes gasosos $\left(\mathrm{C}_{2} \mathrm{H}_{6}+\right.$ vapor de $\left.\mathrm{H}_{2} \mathrm{O}\right)$; $\mathrm{PP}$ - produtos de pirólise. 
O esquema dos processos de pirólise em forno tubular é baseado na abordagem do reator de deslocamento ideal (Fig.1, "zoom" A), que é considerado como um fino pedaço do meio reagente que se move pelo tubo com velocidade $w$, variando os valores $r_{i}, T$ e $P$. A pirólise do etano é descrita por mecanismo de reações elementares que se submetem as leis da cinética química detalhada. É prescrita a dependência da temperatura externa da parede $\left(T_{s}\right)$ ao longo do tubo de serpentina e esta temperatura é determinada por medições experimentais [9] e aproximada por:

$$
T_{s}=a_{s}+b_{s} x^{c_{s}} \quad x=0, \ldots, x_{f}
$$

onde $a_{s}, b_{s}, c_{s}$ são valores constantes.

Neste esquema são considerados também:

- a transferência de calor da parede do tubo à mistura reagente que passa pelo tubo; - a queda de pressão devido à resistência hidrodinâmica;

- a absorção de calor devido às reações endotérmicas.

As hipóteses simplificadas consideradas são: que o fluxo dentro do tubo é unidimensional e estacionário, que as quedas locais de pressão são incluídas nas perdas distribuídas e que as propriedades termofisicas do gás $\lambda_{g}$ e $\eta_{g}$ são constantes.

Apoiando-se nas considerações acima apresentadas, as equações do modelo matemático estão colocadas a seguir.

a) Equações da cinética química na forma exponencial [6]:

$$
\frac{d \gamma_{i}}{d x}=-\frac{1}{w}\left(e^{\gamma_{i}} \sum_{j} v_{i j} \Omega_{j}+\sum_{q} \sum_{j} v_{q j} \Omega_{j}\right) \equiv f_{\gamma_{i}}
$$

onde:

$$
\begin{aligned}
& i=1, \ldots, n_{c} \text { e } j=1, \ldots, m_{c} \\
& \Omega_{j}=k_{j}\left(\frac{P}{R_{o} T}\right)^{\bar{m}_{j}} \exp \left(-\sum_{p} n_{p j} \gamma_{p}\right) ; \bar{m}_{j}=m_{j}+\sum n_{p j}-1 ; \gamma_{i}=-\ln _{i} ; \\
& v_{i j}=v_{i s}^{\prime \prime}-v_{i s}^{\prime} ; n_{i j}=v_{i s}^{\prime} ; j=s ; s=1, \ldots, m_{c} ; \\
& v_{i j}=v_{i s}^{\prime}-v_{i s}^{\prime \prime} ; n_{i j}=v_{i s}^{\prime \prime} ; j=s+m_{c} ; s=1, \ldots, m_{c} ; \\
& v_{i s}^{\prime}, v_{i s}^{\prime \prime} \text { - coeficientes estequiométricos no conjunto da reações reversíveis: }
\end{aligned}
$$

$$
\sum_{i} v_{i s}^{\prime} B_{i} \Longleftrightarrow \sum_{i} v_{i s}^{\prime \prime} B_{i} \quad s=1, \ldots, m_{c}
$$

sendo $B_{i}$ - símbolo da $i$-ésima substância; $m_{j}$ - índice de participação na reação $j$ da partícula catalítica $M\left(m_{j}=1\right.$, se a partícula $M$ participa na $j$-ésima reação e $m_{j}=0$ de maneira oposta).

b) Equação da transferência de calor:

$$
\frac{d Q_{m}}{d x}=\frac{\alpha_{\Sigma} \pi D}{\dot{m}}\left(T_{s}-T\right) \equiv f_{Q}
$$

sendo o coeficiente total de transferência da calor ao fluxo $\left(\alpha_{\Sigma}\right)$ calculado por:

$$
\alpha_{\Sigma}=\lambda_{g} N u / D
$$

com as condições $R e=10^{4}, \operatorname{Pr}=0.7, \ldots, 4$ ([7]) e $N u=0.021 R e^{0.8} \operatorname{Pr}^{0.4}$. 
c) Equação da vazão:

$$
F_{w}=w-\frac{4 \dot{m} R_{o} T}{\pi D^{2} F P \mu_{g}}=0 .
$$

d) Equação de energia que está apresentada conforme [6]:

$$
F_{T} \equiv T-T_{a p}-\sum_{q}\left(\left(h_{o}^{*}+Q_{m}-w^{2} / 2\right) \mu_{q}-H_{q}^{a p}\right) r q / \sum_{q} C_{p q}^{a p} r q=0
$$

onde $q=1, \ldots, n_{c}$.

e) Equação do movimento:

$$
\frac{d P}{d x}=-\frac{P \mu_{g} w}{R_{o} T}\left(\frac{d w}{d x}+\frac{\xi w}{2 D}\right)
$$

sendo o coeficiente de resistência $\xi$, calculado por:

$$
\xi=\frac{0.3165}{R e^{0.25}}+\frac{\sum \xi_{l c}}{x_{f}}
$$

com as condições $R e-10^{4}, \ldots, 10^{6}$ e sendo $\xi_{l c}$ os coeficientes locais de resistência .

Assim, o modelo matemático é constituído pelas equações diferenciais (3.2), (3.4), (3.8) e pelas equações algébricas (3.6) e (3.7), $\operatorname{com} \gamma_{i}, Q_{m}, w, T$ e $P$ grandezas incógnitas.

\section{Método Numérico}

O sistema de equações deste modelo é do tipo "stiff" e para resolvê-lo são aplicados métodos implícitos com uso do Jacobiano ([11]). Mas, a Eq. (3.8), devido à pequena alteração da pressão pode ser integrada pelo esquema explícito (por exemplo, pelo método de Runge-Kutta). Assim, esta equação foi retirada do esquema implícito de resolução e o sistema de equações obtido (Eqs. (3.2), (3.4), (3.6) e (3.7)) resolvido aplicando-se o esquema spline-integração proposto em [5]. Neste esquema as variáveis das equações diferenciais, em cada passo de integração $\left(x_{n}, \ldots, x_{n+1}\right)$, são apresentadas por polinômios quadráticos, a saber:

$$
\gamma_{i}^{n+1}(\overline{\bar{x}})=a_{i}^{n+1}+b_{i}^{n+1} \bar{x}+c_{i}^{n+1} \bar{x}^{2}
$$

onde: $\bar{x}=\left(x-x_{n}\right) / h_{n+1} ; 0 \leq \bar{x} \leq 1 ; \gamma_{i}^{n+1}(0)=\gamma_{i}^{n}$ e $\gamma_{i}^{n+1}(1)=\gamma_{i}^{n+1}$ são valores de $\gamma_{i}$ no início e no fim do passo; $a_{i}^{n+1}, b_{i}^{n+1}$ e $c_{i}^{n+1}$ são coeficientes polinomiais entre os quais somente os coeficientes $c_{i}^{n+1}$ são incógnitas.

Então as equações (3.2) podem ser reescritas na forma:

$$
\frac{d\left(a_{i}^{n+1}+b_{i}^{n+1} \bar{x}+c_{i}^{n+1} \bar{x}^{2}\right)}{d \bar{x}}=h_{n+1} f_{i}\left(<a_{k}^{n+1}+b_{k}^{n+1} \bar{x}+c_{k}^{n+1} \bar{x}^{2}>\right)
$$

onde $h_{n+1}=x_{n+1}-x_{n}$ e $i=1, \ldots, n_{c}$.

Considerando que as equações (4.2) serão satisfeitas nos pontos $x_{n+1}$ (ou seja, $\left.\bar{x}_{n+1}=1\right)$, derivando os seus termos a esquerda e reduzindo os termos a direita, se obtém o sistema: 


$$
F_{i}^{n+1} \equiv b_{i}^{n+1}+2 c_{i}^{n+1}-h_{n+1} f_{i}\left(<c_{k}^{n+1}>\right)=0
$$

que é necessário resolver em cada passo. Na sua resolução é aplicado o esquema de Newton. Os detalhes do método de spline-integração estão apresentados em [5].

\section{Simulações Numéricas}

O modelo matemático dos processos em forno tubular foi realizado pelo código "PIROL" (Fortran Power Station). Simulações numéricas foram executadas para as condições do forno que funciona na empresa "ORGSINTES" [9]. Neste forno a matéria prima (não considerando água) possui a composição: $r_{C_{2} H_{6}}=0.94$; $r_{C_{4}}=0.04 ; r_{C_{3} H_{8}}=0.02$ com parâmetros: $T_{o}=800, \ldots, 850 \mathrm{~K}$, relação $g_{e v}=$ $1, \ldots, 2$ e $P=0.2, \ldots, 0.5 \mathrm{MPa}$. O tubo de serpentina apresenta como dimensões: $x_{f}=83 \mathrm{~m} ; D=0.134 \mathrm{~m}$. A temperatura da parede do tubo (obtida por medições experimentais) foi aproximada por (3.1) com valores: $a_{s}=1000 ; b_{s}=100$ e $c_{s}=$ 0.1835. O mecanismo de reações foi formado a partir da base [8] e é constituído pelas espécies: $\mathrm{H}, \mathrm{H}_{2}, \mathrm{H}_{2} \mathrm{O}, \mathrm{CH}, \mathrm{CH}_{2}, \mathrm{CH}_{3}, \mathrm{CH}_{4}, \mathrm{C}_{2} \mathrm{H}, \mathrm{C}_{2} \mathrm{H}_{2}, \mathrm{C}_{2} \mathrm{H}_{3}, \mathrm{C}_{2} \mathrm{H}_{4}$, $\mathrm{C}_{2} \mathrm{H}_{5}, \mathrm{C}_{2} \mathrm{H}_{6}, \mathrm{C}_{3} \mathrm{H}_{2}, \mathrm{C}_{3} \mathrm{H}_{4}, \mathrm{C}_{3} \mathrm{H}_{5}, \mathrm{C}_{3} \mathrm{H}_{6}, \mathrm{C}_{3} \mathrm{H}_{7}, \mathrm{C}_{3} \mathrm{H}_{8}$ e as reações selecionadas estão dadas na Tabela 1 .

A distribuição das características do fluxo e a comparação com dados experimentais no regime do funcionamento (com parâmetros: $T_{o}=836 \mathrm{~K}, P=0.375$ $\mathrm{MPa}, \dot{m}=0.825 \mathrm{~kg}$ e relação $\left.g_{e v}=1.71\right)$ estão mostradas nas Fig. 2 e 3 .

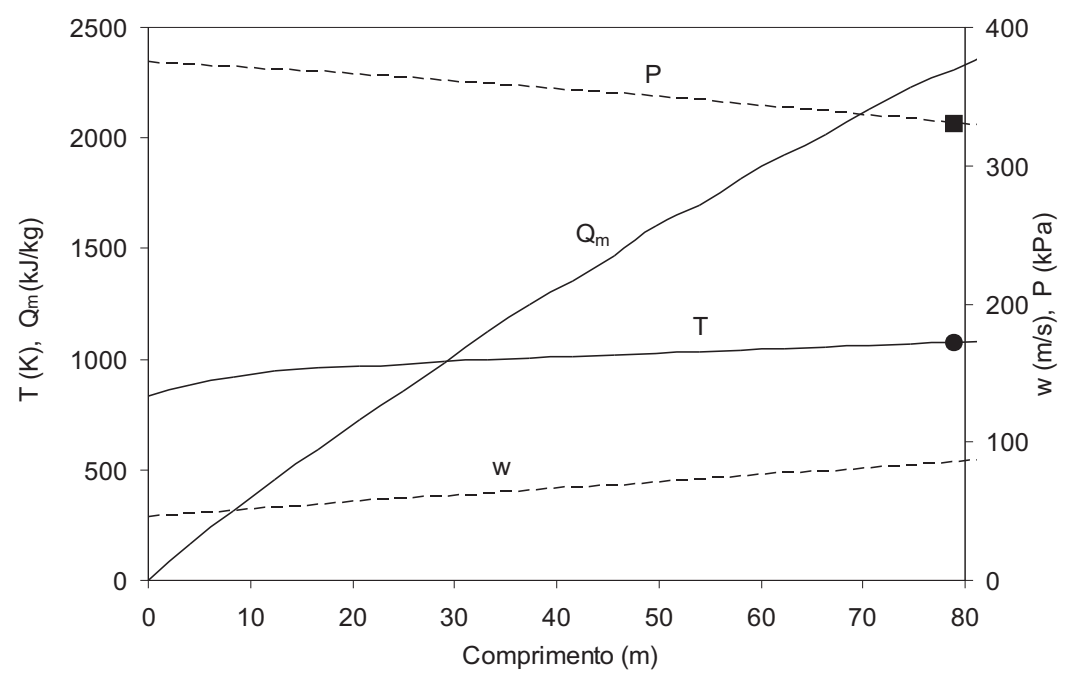

Figura 2: Alteração das características do fluxo $P, Q_{m}, T$ e $w$ ao longo do tubo (• e - dados experimentais).

A Figura 2 apresenta as alterações das características do fluxo: $P, Q_{m}, T$, $w=f(x)$. Como é visível a pressão sofre pequenas alterações de $0.375 \mathrm{MPa}$ até 
Tabela 1: Mecanismo de pirólise do etano.

\begin{tabular}{|c|c|c|c|}
\hline$N$ & Reação & $N$ & Reação \\
\hline 1 & $H+H+M=H_{2}+M$ & 2 & $\mathrm{CH}_{3}+\mathrm{M}=\mathrm{CH}+\mathrm{H}_{2}+\mathrm{M}$ \\
\hline 3 & $\mathrm{CH}_{2}+\mathrm{H}=\mathrm{CH}+\mathrm{H}_{2}$ & 4 & $\mathrm{CH}+\mathrm{C}_{2} \mathrm{H}_{2}=\mathrm{C}_{3} \mathrm{H}_{2}+\mathrm{H}$ \\
\hline 5 & $\mathrm{CH}_{4}+\mathrm{M}=\mathrm{CH}_{3}+\mathrm{H}+\mathrm{M}$ & 6 & $\mathrm{CH}_{4}+\mathrm{H}=\mathrm{CH}_{3}+\mathrm{H}_{2}$ \\
\hline 7 & $\mathrm{CH}_{4}+\mathrm{CH}_{2}=\mathrm{CH}_{3}+\mathrm{CH}_{3}$ & 8 & $\mathrm{CH}_{3}+\mathrm{M}=\mathrm{CH}_{2}+\mathrm{H}+\mathrm{M}$ \\
\hline 9 & $\mathrm{CH}_{3}+\mathrm{H}=\mathrm{CH}_{2}+\mathrm{H}_{2}$ & 10 & $\mathrm{CH}_{2}+\mathrm{H}=\mathrm{CH}+\mathrm{H}_{2}$ \\
\hline 11 & $\mathrm{CH}_{2}+\mathrm{CH}=\mathrm{C}_{2} \mathrm{H}_{2}+\mathrm{H}$ & 12 & $\mathrm{CH}_{2}+\mathrm{CH}_{2}=\mathrm{C}_{2} \mathrm{H}_{2}+\mathrm{H}_{2}$ \\
\hline 13 & $\mathrm{CH}+\mathrm{H}=\mathrm{C}+\mathrm{H}_{2}$ & 14 & $\mathrm{CH}+\mathrm{CH}_{3}=\mathrm{C}_{2} \mathrm{H}_{3}+\mathrm{H}$ \\
\hline 15 & $\mathrm{CH}+\mathrm{CH}_{4}=\mathrm{C}_{2} \mathrm{H}_{4}+\mathrm{H}$ & 16 & $\mathrm{CH}+\mathrm{C}_{2} \mathrm{H}_{4}=\mathrm{CH}_{2}+\mathrm{C}_{2} \mathrm{H}_{3}$ \\
\hline 17 & $\mathrm{CH}+\mathrm{C}_{2} \mathrm{H}_{6}=\mathrm{C}_{2} \mathrm{H}_{4}+\mathrm{CH}_{3}$ & 18 & $\mathrm{C}+\mathrm{CH}_{4}=\mathrm{CH}+\mathrm{CH}_{3}$ \\
\hline 19 & $\mathrm{C}+\mathrm{CH}_{3}=\mathrm{C}_{2} \mathrm{H}_{2}+\mathrm{H}$ & 20 & $\mathrm{C}+\mathrm{CH}_{2}=\mathrm{C}_{2} \mathrm{H}+\mathrm{H}$ \\
\hline 21 & $\mathrm{CH}_{3}+\mathrm{CH}_{3}=\mathrm{C}_{2} \mathrm{H}_{6}$ & 22 & $\mathrm{C}_{2} \mathrm{H}_{5}+\mathrm{H}=\mathrm{CH}_{3}+\mathrm{CH}_{3}$ \\
\hline 23 & $\mathrm{CH}_{3}+\mathrm{CH}_{3}=\mathrm{C}_{2} \mathrm{H}_{4}+\mathrm{H}_{2}$ & 24 & $\mathrm{CH}_{3}+\mathrm{CH}_{2}=\mathrm{C}_{2} \mathrm{H}_{4}+\mathrm{H}$ \\
\hline 25 & $\mathrm{C}_{2} \mathrm{H}_{6}+\mathrm{H}=\mathrm{C}_{2} \mathrm{H}_{5}+\mathrm{H}_{2}$ & 26 & $\mathrm{C}_{2} \mathrm{H}_{5}+\mathrm{H}=\mathrm{C}_{2} \mathrm{H}_{4}+\mathrm{H}_{2}$ \\
\hline 27 & $\mathrm{C}_{2} \mathrm{H}_{5}+\mathrm{H}=\mathrm{C}_{2} \mathrm{H}_{6}$ & 28 & $\mathrm{C}_{2} \mathrm{H}_{4}+\mathrm{CH}_{3}=\mathrm{C}_{2} \mathrm{H}_{3}+\mathrm{CH}_{4}$ \\
\hline 29 & $\mathrm{C}_{2} \mathrm{H}_{6}+\mathrm{CH}_{3}=\mathrm{C}_{2} \mathrm{H}_{5}+\mathrm{CH}_{4}$ & 30 & $\mathrm{C}_{2} \mathrm{H}_{6}+\mathrm{CH}_{2}=\mathrm{CH}_{3}+\mathrm{C}_{2} \mathrm{H}_{5}$ \\
\hline 31 & $\mathrm{C}_{2} \mathrm{H}_{5}+M=\mathrm{C}_{2} \mathrm{H}_{4}+\mathrm{H}+\mathrm{M}$ & 32 & $\mathrm{C}_{2} \mathrm{H}_{4}+\mathrm{M}=\mathrm{C}_{2} \mathrm{H}_{2}+\mathrm{H}_{2}+M$ \\
\hline 33 & $\mathrm{C}_{2} \mathrm{H}_{4}+M=\mathrm{C}_{2} \mathrm{H}_{3}+H+M$ & 34 & $\mathrm{C}_{2} \mathrm{H}_{4}+\mathrm{H}=\mathrm{C}_{2} \mathrm{H}_{3}+\mathrm{H}_{2}$ \\
\hline 35 & $\mathrm{C}_{2} \mathrm{H}_{3}+M=\mathrm{C}_{2} \mathrm{H}_{2}+\mathrm{H}+\mathrm{M}$ & 36 & $\mathrm{C}_{2} \mathrm{H}_{3}+\mathrm{H}=\mathrm{C}_{2} \mathrm{H}_{2}+\mathrm{H}_{2}$ \\
\hline 37 & $\mathrm{C}_{2} \mathrm{H}_{3}+\mathrm{CH}_{2}=\mathrm{C}_{2} \mathrm{H}_{2}+\mathrm{CH}_{3}$ & 38 & $\mathrm{C}_{2} \mathrm{H}_{3}+\mathrm{C}_{2} \mathrm{H}=\mathrm{C}_{2} \mathrm{H}_{2}+\mathrm{C}_{2} \mathrm{H}_{2}$ \\
\hline 39 & $\mathrm{C}_{2} \mathrm{H}_{3}+\mathrm{CH}=\mathrm{CH}_{2}+\mathrm{C}_{2} \mathrm{H}_{2}$ & 40 & $\mathrm{C}_{2} \mathrm{H}_{3}+\mathrm{CH}_{3}=\mathrm{C}_{3} \mathrm{H}_{5}+\mathrm{H}$ \\
\hline 41 & $\mathrm{C}_{2} \mathrm{H}_{3}+\mathrm{CH}_{3}=\mathrm{C}_{2} \mathrm{H}+\mathrm{CH}_{4}$ & 42 & $\mathrm{C}_{2} \mathrm{H}_{2}+\mathrm{CH}_{3}=\mathrm{C}_{2} \mathrm{H}+\mathrm{CH}_{4}$ \\
\hline 43 & $\mathrm{C}_{2} \mathrm{H}_{2}+M=\mathrm{C}_{2} \mathrm{H}+\mathrm{H}+\mathrm{M}$ & 44 & $\mathrm{C}_{2} \mathrm{H}+\mathrm{H}_{2}=\mathrm{C}_{2} \mathrm{H}_{2}+\mathrm{H}$ \\
\hline 45 & $\mathrm{C}_{3} \mathrm{H}_{8}=\mathrm{C}_{2} \mathrm{H}_{5}+\mathrm{CH}_{3}$ & 46 & $\mathrm{C}_{3} \mathrm{H}_{8}+\mathrm{H}=\mathrm{C}_{3} \mathrm{H}_{7}+\mathrm{H}_{2}$ \\
\hline 47 & $\mathrm{C}_{3} \mathrm{H}_{8}+\mathrm{CH}_{3}=\mathrm{C}_{3} \mathrm{H}_{7}+\mathrm{CH}_{4}$ & 48 & $\mathrm{C}_{3} \mathrm{H}_{8}+\mathrm{C}_{2} \mathrm{H}_{3}=\mathrm{C}_{3} \mathrm{H}_{7}+\mathrm{C}_{2} \mathrm{H}_{4}$ \\
\hline 49 & $\mathrm{C}_{3} \mathrm{H}_{8}+\mathrm{C}_{2} \mathrm{H}_{5}=\mathrm{C}_{3} \mathrm{H}_{7}+\mathrm{C}_{2} \mathrm{H}_{6}$ & 50 & $\mathrm{C}_{3} \mathrm{H}_{8}+\mathrm{C}_{3} \mathrm{H}_{5}=\mathrm{C}_{3} \mathrm{H}_{6}+\mathrm{C}_{3} \mathrm{H}_{7}$ \\
\hline 51 & $\mathrm{C}_{3} \mathrm{H}_{7}=\mathrm{C}_{2} \mathrm{H}_{4}+\mathrm{CH}_{3}$ & 52 & $\mathrm{C}_{3} \mathrm{H}_{6}+\mathrm{H}=\mathrm{C}_{3} \mathrm{H}_{7}$ \\
\hline 53 & $\mathrm{C}_{3} \mathrm{H}_{7}+\mathrm{H}=\mathrm{C}_{2} \mathrm{H}_{5}+\mathrm{CH}_{3}$ & 54 & $\mathrm{C}_{3} \mathrm{H}_{6}=\mathrm{C}_{2} \mathrm{H}_{2}+\mathrm{CH}_{4}$ \\
\hline 55 & $\mathrm{C}_{3} H_{6}=\mathrm{C}_{3} H_{4}+H_{2}$ & 56 & $\mathrm{C}_{3} \mathrm{H}_{5}+\mathrm{H}=\mathrm{C}_{3} \mathrm{H}_{6}$ \\
\hline 57 & $\mathrm{C}_{3} \mathrm{H}_{6}+\mathrm{H}=\mathrm{C}_{2} \mathrm{H}_{4}+\mathrm{CH}_{3}$ & 58 & $\mathrm{C}_{3} \mathrm{H}_{6}+\mathrm{H}=\mathrm{C}_{3} \mathrm{H}_{5}+\mathrm{H}_{2}$ \\
\hline 59 & $\mathrm{C}_{3} \mathrm{H}_{6}+\mathrm{CH}_{3}=\mathrm{C}_{3} \mathrm{H}_{5}+\mathrm{CH}_{4}$ & 60 & $\mathrm{C}_{3} \mathrm{H}_{5}+\mathrm{H}=\mathrm{C}_{3} \mathrm{H}_{4}+\mathrm{H}_{2}$ \\
\hline 61 & $\mathrm{C}_{3} \mathrm{H}_{5}+\mathrm{CH}_{3}=\mathrm{C}_{3} \mathrm{H}_{4}+\mathrm{CH}_{4}$ & 62 & $\mathrm{C}_{3} \mathrm{H}_{4}+\mathrm{H}=\mathrm{CH}_{3}+\mathrm{C}_{2} \mathrm{H}_{2}$ \\
\hline 63 & $\mathrm{C}_{3} \mathrm{H}_{4}+\mathrm{H}=\mathrm{C}_{3} \mathrm{H}_{5}$ & & \\
\hline
\end{tabular}

$0.325 \mathrm{MPa}$ e a velocidade cresce de $45 \mathrm{~m} / \mathrm{s}$ até $85 \mathrm{~m} / \mathrm{s}$. A temperatura cresce com aproximadamente $\Delta T=250 \mathrm{~K}$. Ao mesmo tempo o calor absorvido pelo meio reagente é grande: $Q_{m}^{f}=2250 \mathrm{~kJ} / \mathrm{kg}$. Se a composição não fosse alterada o calor específico médio seria: $\bar{C}_{p}=2250 / 250 \approx 10 \mathrm{~kJ} / \mathrm{kgK}$. Mas, os calores específicos de cada espécie não superam os valores: $C_{p} \approx 1.5 \mathrm{~kJ} / \mathrm{kgK}$. Este efeito ocorre devido a reações endotérmicas que exigem grandes gastos de energia.

$\mathrm{Na}$ Figura 3 são apresentadas as evoluções das principais espécies: $\mathrm{H}_{2}, \mathrm{H}_{2} \mathrm{O}$, $\mathrm{CH}_{4}, \mathrm{C}_{2} \mathrm{H}_{4}$ e $\mathrm{C}_{2} \mathrm{H}_{6}$ ao longo do tubo e também resultados experimentais na saída 


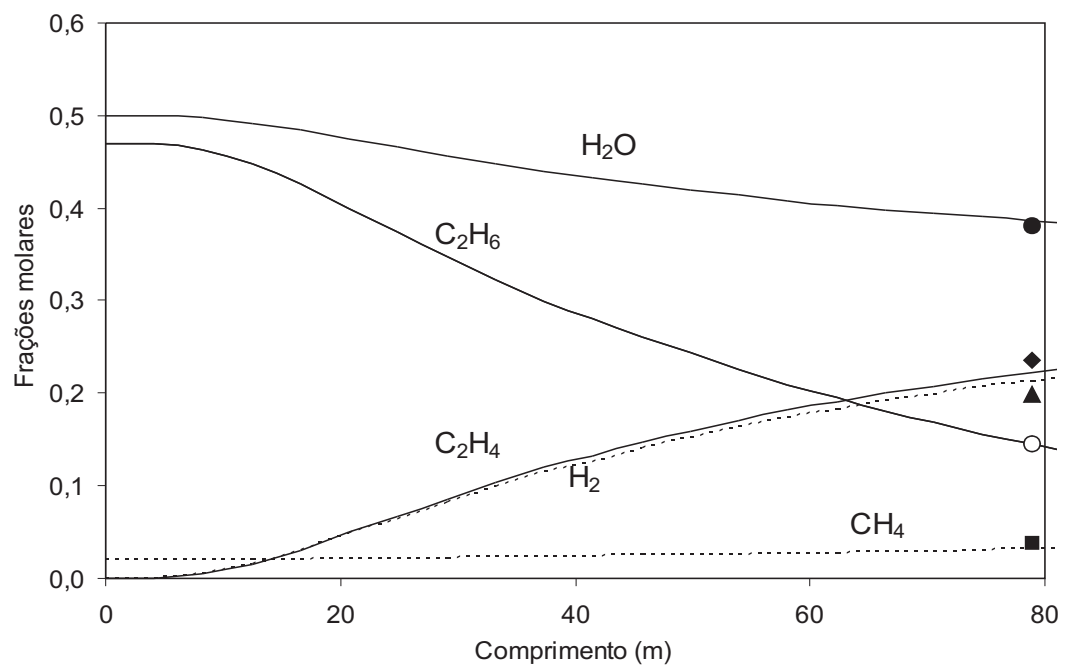

Figura 3: Alteração das frações molares das espécies $\mathrm{H}_{2}, \mathrm{H}_{2} \mathrm{O}, \mathrm{CH}_{4}, \mathrm{C}_{2} \mathrm{H}_{4}$ e $\mathrm{C}_{2} \mathrm{H}_{6}$, ao longo do tubo de serpentina (dados experimentais: $\bullet-\mathrm{H}_{2} \mathrm{O}, \mathbf{\square}-\mathrm{CH}_{4}, \circ-\mathrm{C}_{2} \mathrm{H}_{4}$, $\checkmark-\mathrm{C}_{2} \mathrm{H}_{6}$ e $\left.\boldsymbol{\Delta}-\mathrm{H}_{2}\right)$.

do forno. É observada uma boa concordância com os resultados numéricos (erro relativo $\approx 8 \%$ ). $\mathrm{O}$ vapor de água não participa de nenhuma reação, mas a sua fração molar $\left(r_{\mathrm{H}_{2} \mathrm{O}}\right)$ diminui devido a decomposição do $\mathrm{C}_{2} H_{6}$ (a fração mássica do vapor de água não é alterada).

Como é visível a fração mássica do etano $\left(r_{C_{2} H_{6}}\right)$ é essencialmente reduzida (de $47 \%$ até $13 \%$ ) num pequeno intervalo de temperatura. Ao mesmo tempo as concentrações de $\mathrm{C}_{2} \mathrm{H}_{4}$ e $\mathrm{H}_{2}$ são formadas em quantias consideráveis e quase iguais $\left(r_{C_{2} H_{4}}^{f}=0.23 ; r_{H_{2}}^{f}=0.22\right)$. Uma superação de $r_{C_{2} H_{4}}^{f}$ sob $r_{H_{2}}^{f}$ é causada pela formação do metano $\left(r_{C H_{4}}^{f}=0.08\right)$ que é uma espécie concomitante a pirólise. É conhecido ([1], [9]) que o metano é uma preocupação constante dos engenheiros que projetam e exploram fornos para a pirólise do etano, dessa forma, o modelo elaborado pode ajudar na escolha dos parâmetros ótimos do processo de pirólise.

Por exemplo, com a alteração da capacidade térmica dos maçaricos (Fig.1) é alterada também a temperatura das telas irradiantes e, por conseguinte $T_{s}$, o que influi no processo de pirólise. Este efeito pode ser simulado pela alteração do termo $a_{s}=T_{\text {so }}$ na Eq. 3.1. Estes cálculos foram realizados no intervalo $T_{\text {so }}=800, \ldots, 1200$ $\mathrm{K}$ deixando os outros parâmetros iguais ao regime básico $\left(T_{s o}=1000 \mathrm{~K}\right)$.

Nas Figuras 4 e 5 são mostradas as características da pirólise na saída do forno em função de $T_{s o}$. Pode-se observar que:

- a pressão na saída $\left(P_{f}\right)$ é quase constante;

- a alteração da temperatura na parede do tubo em todo intervalo $\Delta T_{s o}=400 \mathrm{~K}$, provoca alteração na temperatura $T_{f}$ no intervalo $\Delta T_{f}=300 \mathrm{~K}$, pela ocorrência de reações endotérmicas;

- na região onde $T_{s o}=800, \ldots, 950 \mathrm{~K}$, o forno funciona com rendimento baixo, ou 


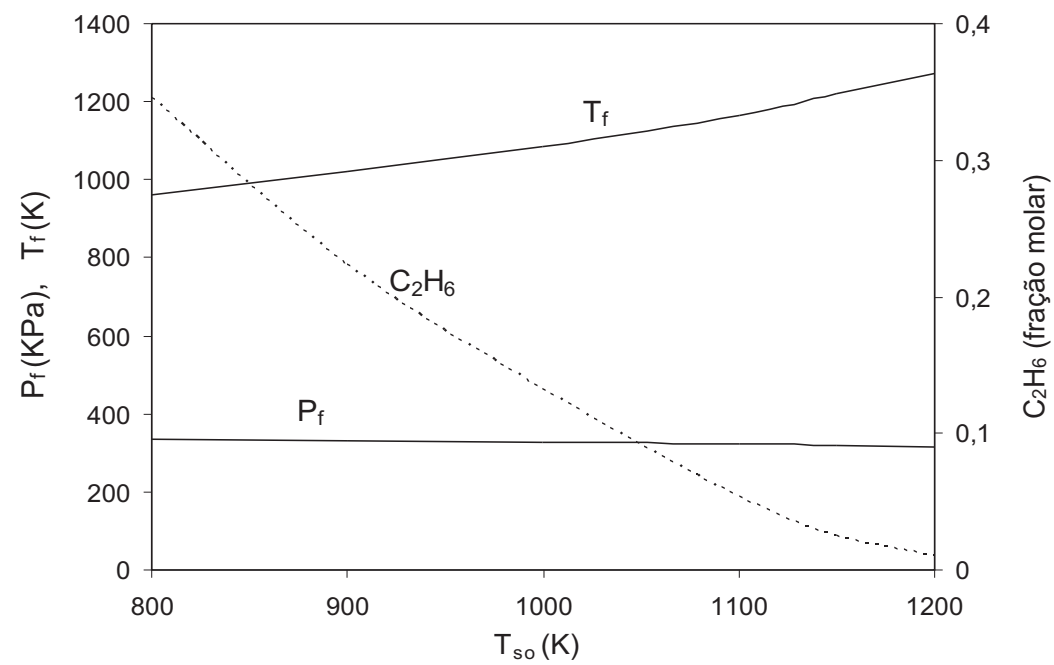

Figura 4: Alteração das características $P_{f}, T_{f}$ e $r_{C_{2} H_{6}}^{f}$ em função de $T_{s o}=a_{s}$.

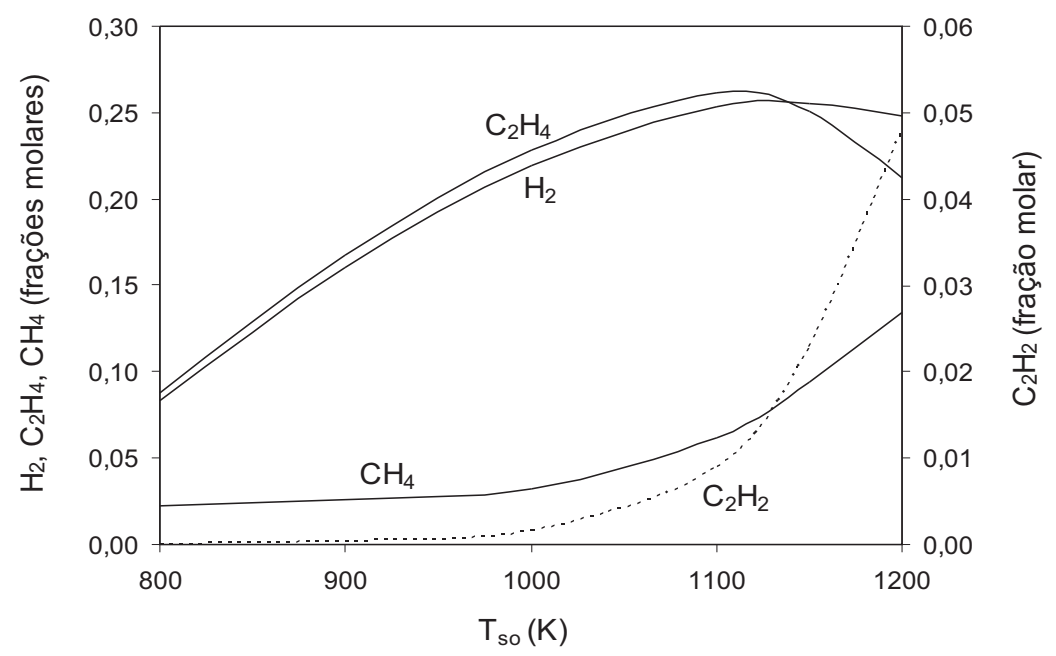

Figura 5: Alteração das características das espécies $r_{H_{2}}^{f}, r_{C H_{4}}^{f}, r_{C_{2} H_{4}}^{f}, r_{C_{2} H_{2}}^{f}$ em função de $T_{\text {so }}$.

seja, $r_{C_{2} H_{4}}^{f} r_{C_{2} H_{6}}^{f}=0.2, \ldots, 1$ e as substâncias concomitantes $\left(C_{4}\right.$ e $\left.C_{2} H_{2}\right)$ não são formadas;

- na região $T_{s o}=1000, \ldots, 1100 \mathrm{~K}$ o forno tem um bom funcionamento $\left(r_{C_{2} H_{4}}^{f} r_{C_{2} H_{6}}^{f}=\right.$ $1.4, \ldots, 4)$ gerando pequenas concentrações das substâncias $\mathrm{CH}_{4}$ e $\mathrm{C}_{2} \mathrm{H}_{2}\left(r_{\mathrm{CH}_{4}}^{f} \leq\right.$ 0.05 e $r_{C_{2} H_{2}}^{f} \leq 0.01$ ); 
- para $T_{s o}>1100 \mathrm{~K}$ a produtividade do forno diminui, ou seja, $r_{C_{2} H_{4}}^{f}$ começa a ser reduzido (de 0.25 a 0.2 ) apesar do etano quase desaparecer. Este efeito é explicado pelo crescimento do metano $\left(r_{C H_{4}}^{f} \approx 0.12\right)$ e principalmente do acetileno $\left(r_{C_{2} H_{2}}^{f} \approx 0.05\right)$. Assim este regime de trabalho do forno não é desejável.

\title{
6. Conclusões
}

A pirólise do etano é amplamente utilizada na indústria petroquímica para produzir o etileno. Este processo é complexo e a sua modelagem matemática é um problema bastante atual. Neste trabalho foi elaborado um modelo matemático para descrever o processo de pirólise em forno tubular. O modelo matemático é baseado no esquema do reator de deslocamento ideal e considera: decomposição do etano por mecanismo de reações elementares; transferência de calor ao meio reagente e a queda de pressão pela resistência hidrodinâmica.

Para resolver as equações diferenciais do tipo "stiff" foi aplicado o método de spline-integração com o esquema implícito. Simulações numéricas foram realizadas pelo código "PIROL" para as condições de funcionamento do forno industrial. Os resultados correspondem aos dados experimentais obtidos na saída do forno (com erro relativo de $\approx 8 \%$ ). As simulações numéricas com a alteração de temperatura da parede do tubo de serpentina mostram a forte influência deste fator nas características da produtividade. O modelo desenvolvido pode ser usado para projetos de forno industrial.

\begin{abstract}
Mathematical model for the ethane pyrolysis process in coil tubular furnace is proposed. The reacting system is described by the mechanism that includes the 63 chemical reactions and 19 species. It is also considered the changes of pressure, temperature, flow velocity and heat absorption by endothermic reactions. To solve the resulting system of equations, it is used the spline-integration method with implicit scheme. The simulations were performed for the operating conditions of industrial furnace. The numerical simulations for these conditions had shown the good agreement with experimental data. The computational results correctly are predicted the physical-chemical phenomena, observed in furnace and the developed model can be used for its design.
\end{abstract}

Keywords. Ethane, Pyrolysis, Mathematical Model, Tubular Coil, Reactions Mechanism.

\section{Referências}

[1] L.F. Albright, B.F. Cruns and W.H. Corcoran, Pyrolysis: theory and industrial practice, Academic press, New York, 1983.

[2] M. Berren, M. Wang, Modelling and dynamic optimization of termal cracking manufacturing, Computers e Chemical Engineering, 35, No. 12 (2011), 28762885 . 
[3] M.H. Buffenour, J.M. Aubry and X. Hurstel, Large ethylene plants present unique design construction challenges, Oil and Gas Journal, 102, No. 3 (2004), 60-65.

[4] R. Kazimzadeh, H.R. Godini and M. Chashghace, Flowsheeting of steam crachihg furnace, Chemical Engineering Research and Disign, 87, No. 1 (2009),3646.

[5] V.G. Krioukov, R.L. Iskhakova, A. Durigon, J.C.R. Claeyssen, Application of the method of spline-integration for the modeling of combustion processes, in Proceedings of XXIV Iberian Latin-American Congress on Computational Methods in Engineering, pp. 1-15, Ouro Preto, 2003.

[6] V.G. Krioukov, V.I. Naomov, A.L. Abdullin, T.V. Trinos, "Combustion and flow in propulsion and power generation systems: modeling, energy, ecology", Yanus, Moscow, 1997.

[7] A.I. Leonthev, Teoria da transporte de calor e de massa. Escola superior, Moscow, 1975.

[8] N.M. Marinov, W.J. Pitz, C.K. Westbrook, C.F. Melius, Aromatic and polycyclic aromatic hydrocarbon formation in a laminar premixed n- butane flame,Combustion and Flame, 114 (1998), 192-213.

[9] ORGSINTES, "Produção de etileno de 4 grau: regulamento tecnológico", Empresa ORGSINTES, Kazan, Rússia, 1986.

[10] C. Riverol, M.V. Polipovik, Optimization of the pyrolysis of ethane using fuzzy programming, Chemical Engineering Journal, bf 133, No. 1-3 (2007), 133 - 137.

[11] A.P. Spilimbergo, A.V. Fafurin, V.G. Krioukov, Modelagem matemática da Pirólise do etano empPlanta industrial, in Anais do XXXII Congresso Nacional de Matemática Aplicada e Computacional - CNMAC, pp. 1-7, Águas de Lindóia, 2010. 